\title{
FINITE LOGARITHMIC ORDER SOLUTIONS OF LINEAR $q$-DIFFERENCE EQUATIONS
}

\author{
ZHI-TAO Wen
}

Abstract. During the last decade, several papers have focused on linear $q$-difference equations of the form

$$
\sum_{j=0}^{n} a_{j}(z) f\left(q^{j} z\right)=a_{n+1}(z)
$$

with entire or meromorphic coefficients. A tool for studying these equations is a $q$-difference analogue of the lemma on the logarithmic derivative, valid for meromorphic functions of finite logarithmic order $\rho_{\log }$. It is shown, under certain assumptions, that $\rho_{\log }(f)=\max \left\{\rho_{\log }\left(a_{j}\right)\right\}+1$. Moreover, it is illustrated that a $q$-Casorati determinant plays a similar role in the theory of linear $q$-difference equations as a Wronskian determinant in the theory of linear differential equations. As a consequence of the main results, it follows that the $q$-gamma function and the $q$-exponential functions all have logarithmic order two.

\section{Introduction}

Recently, many papers have focused on linear $q$-difference equations of the form

$$
\sum_{j=0}^{n} a_{j}(z) f\left(q^{j} z\right)=a_{n+1}(z),
$$

where $q \in \mathbb{C}, 0<|q|<1$ and $a_{j}(z)(j=1, \ldots, n+1)$ are rational functions. In [5], Bergweiler and Hayman study the special case

$$
f(z)-a(z) f(c z)=0,
$$

where $0<|c|<1$ and $a(z)$ is a polynomial of degree $d$. They show that if $d=0$ (so that $a(z)$ is a constant), then the only solutions of (2) are monomials

Received September 20, 2012.

2010 Mathematics Subject Classification. Primary 39A13; Secondary 30D35.

Key words and phrases. logarithmic Borel exceptional value, logarithmic derivative, logarithmic exponent of convergence, logarithmic order, $q$-Casorati determinant, $q$-difference equation.

This research was supported in part by China Scholarship Council(CSC). 
$f(z)=A z^{p}$. In particular, there are no transcendental solutions. If $d>0$, then there exists a nonnegative integer $p$ and $A, z_{1}, \ldots, z_{d}$ in $\mathbb{C} \backslash\{0\}$ such that

$$
f(z)=A z^{p} \prod_{\mu=1}^{d} \prod_{j=0}^{\infty}\left(1-\frac{c^{j} z}{z_{\mu}}\right) .
$$

Evidently the function $f$ given by (3) satisfies (2) for

$$
a(z)=c^{-p} \prod_{\mu=1}^{d}\left(1-\frac{z}{z_{\mu}}\right) .
$$

In [6], Bergweiler et al. treat (1). They conclude that all meromorphic solutions $f$ of $(1)$ satisfy $T(r, f)=O\left((\log r)^{2}\right)$, and that all transcendental meromorphic solutions of (1) satisfy $(\log r)^{2}=O(T(r, f))$. Moreover, if all coefficients of (1) are constants, then all solutions are rational. Later on, many papers go further in the study of linear $q$-difference equations with rational coefficients (see [7]) or meromorphic coefficients (see $[8,9]$ ).

In studying zero-order meromorphic functions, we make use of the concept of logarithmic order [11]. An increasing function $\phi(r)$ is said to be of logarithmic order $\rho$ if

$$
\limsup _{r \rightarrow \infty} \frac{\log ^{+} \phi(r)}{\log \log r}=\rho .
$$

The logarithmic order of a meromorphic function $f(z)$ in $\mathbb{C}$ is defined as the logarithmic order of its characteristic function $T(r, f)$, and denoted by $\rho_{\log }(f)$. If $f$ is a meromorphic function of finite logarithmic order, then $f$ is of usual order zero. Any rational function is of logarithmic order one, however, a function of logarithmic order one is not necessarily a rational function. Constant functions have zero logarithmic order, while there are no meromorphic functions of logarithmic order between zero and one.

An entire function of finite logarithmic order must be a canonical product, and hence a meromorphic function is a ratio of two canonical products. If $\phi(r)$ is an increasing function and convex in $\log r$ such that $\phi(r) \neq O(\log r)$, then an entire function $f$ can be constructed such that $\log M(r, f) \sim \phi(r)$, $T(r, f) \sim \phi(r)$ and $N(r, 1 /(f-c)) \sim \phi(r)$ for any $c \in \mathbb{C}[12,13]$. In particular, for any $\rho \in(1, \infty)$ there exists an entire function $f$ such that $\rho_{\log }(f)=\rho$.

The aim of this paper is to study the solutions of $q$-difference equations in terms of logarithmic order. One of the main results is stated as follows.

Theorem 1.1. Let $a_{0}(z), \ldots, a_{n}(z)$ be entire functions of finite logarithmic order, and let $q \in \mathbb{C} \backslash\{0\}$ be such that $|q| \neq 1$. If $f$ is a meromorphic solution of

$$
\sum_{j=0}^{n} a_{j}(z) f\left(q^{j} z\right)=0
$$


and if there exists an integer $i \in\{0, \ldots, n\}$ such that

$$
\rho_{\log }\left(a_{i}\right)>\max _{j \neq i}\left\{\rho_{\log }\left(a_{j}\right)\right\},
$$

then $\rho_{\log }(f)=\rho_{\log }\left(a_{i}\right)+1$.

In [6], Bergweiler shows that all transcendental meromorphic solutions of (1) is of logarithmic order two. Theorem 1.1 extends the rational coefficients to any finite logarithmic order coefficients.

The reminder of this paper is organized as follows. In Section 2, we obtain a $q$-difference analogue for the lemma on the logarithmic derivative. This tool will be applied frequently in the further sections. In Section 3, we consider the logarithmic exponent of convergence for the a-points of a meromorphic function. We obtain, for example, a growth estimate for $\log 1 /|P(z)|$, where $P(z)$ is entire and of finite logarithmic order. In Section 4 , we show that a $q$-Casorati determinant plays a similar role in the theory of $q$-difference equations as a Wronskian determinant in the theory of linear differential equations. After these preparations, we are ready to prove Theorem 1.1 in Section 5. Some further remarks concerning first and second order $q$-difference equations are given in Section 6. Finally, three examples are given in Section 7. In particular, Theorem 1.1 is used to show that the $q$-gamma function and the $q$-exponential functions have logarithmic order two. Moreover, we give an elementary algorithm for constructing entire functions of any pre-given integer logarithmic order.

\section{2. $q$-difference logarithmic derivative analogue}

When working with $q$-difference equations, we need a $q$-difference analogue of

$$
m\left(r, \frac{f^{\prime}}{f}\right)=O(\log r T(r, f))=S(r, f),
$$

for meromorphic functions of finite logarithmic order. This estimate holds without an exceptional set, and is based on the following lemma.

Lemma 2.1 ([3], Lemma 5.1). Let $f$ be a meromorphic function such that $f(0) \neq 0, \infty$, and let $q \in \mathbb{C} \backslash\{0\}$. Then

$$
\begin{aligned}
m\left(r, \frac{f(q z)}{f(z)}\right) \leq & \left(n(\lambda, f)+n\left(\lambda, \frac{1}{f}\right)\right) \\
& \left(\frac{|q-1|^{\delta}\left(|q|^{\delta}+1\right)}{\delta(1-\delta)|q|^{\delta}}+\frac{|q-1| r}{\lambda-|q| r}+\frac{|q-1| r}{\lambda-r}\right) \\
& +\frac{4|q-1| r \lambda}{(\lambda-r)(\lambda-|q| r)}\left(T(\lambda, f)+\log ^{+}\left|\frac{1}{f(0)}\right|\right)
\end{aligned}
$$

where $z=r e^{i \phi}, \lambda>\max \{r,|q| r\}$ and $0<\delta<1$. 
Theorem 2.2. Let $f$ be a meromorphic function of finite logarithmic order $\rho_{\log }(f)$ such that $f(0) \neq 0, \infty$, and let $q \in \mathbb{C} \backslash\{0\}$. Then

$$
m\left(r, \frac{f(q z)}{f(z)}\right)=O\left((\log r)^{\rho_{\log }(f)-1+\varepsilon}\right)
$$

for each $\varepsilon>0$.

Proof. For every $\varepsilon>0$ there exists an $R>0$ such that

$$
T(r, f)=O\left((\log r)^{\rho_{\log }(f)+\varepsilon}\right), \quad r \geq R .
$$

By the definition of $N(r, f)$, we deduce that

$$
N\left(r^{2}, f\right)-N(r, f)=\int_{r}^{r^{2}} \frac{n(r, f)}{t} d t \geq n(r, f) \int_{r}^{r^{2}} \frac{d t}{t} .
$$

Thus,

$$
n(r, f) \leq \frac{N\left(r^{2}, f\right)}{\log r} \leq \frac{T\left(r^{2}, f\right)}{\log r}=O\left((\log r)^{\rho_{\log }(f)-1+\varepsilon}\right) .
$$

Similarly,

$$
n\left(r, \frac{1}{f}\right)=O\left((\log r)^{\rho_{\log }(f)-1+\varepsilon}\right) .
$$

Finally, we choose $\lambda=r^{2}$ in Lemma 2.1, and make use of (5) and (6). This proves the assertion.

Corollary 2.3. Let $f$ be a meromorphic function of finite logarithmic order $\rho_{\log }(f)$ such that $f(0) \neq 0, \infty$, and let $q_{1}, q_{2} \in \mathbb{C} \backslash\{0\}$. Then

$$
m\left(r, \frac{f\left(q_{1} z\right)}{f\left(q_{2} z\right)}\right)=O\left((\log r)^{\rho_{\log }(f)-1+\varepsilon}\right)
$$

for each $\varepsilon>0$.

\section{Logarithmic exponent of convergence}

Let $f$ be meromorphic in $\mathbb{C}$, and let $\left\{z_{n}(a)\right\}$ be the sequence of $a$-points of $f$, where $a \in \mathbb{C} \bigcup\{\infty\}$. Following [11], the logarithmic exponent of convergence of the sequence $\left\{z_{n}(a)\right\}$ is given by

$$
\lambda_{\log }(f, a)=\inf \left\{\mu>0: \sum_{n=1}^{\infty} 1 /\left.|\log | z_{n}(a)\right|^{\mu}<\infty\right\} .
$$

We denote $\lambda_{\log }(f)=\lambda_{\log }(f, 0)$ for short.

Lemma 3.1 ([11]). Let $f$ be a meromorphic function of finite logarithmic order, and let $a \in \mathbb{C}$. Then the logarithmic order of $n(r, 1 /(f-a))$ equals to $\lambda_{\log }(f, a)$. Moreover, $N(r, 1 /(f-a))$ is of logarithmic order $\lambda_{\log }(f, a)+1$. 
By the second fundamental theorem, the characteristic $T(r, f)$ of a transcendental meromorphic function $f$ is typically dominated by three integrated counting functions. If $f$ is of finite logarithmic order, then $T(r, f)$ can be dominated by two integrated counting functions.

Lemma 3.2 ([11], Theorem 7.1). If $f$ is a transcendental meromorphic function of finite logarithmic order, then for any two distinct extended complex values $a$ and $b$, we have

$$
T(r, f) \leq N\left(r, \frac{1}{f-a}\right)+N\left(r, \frac{1}{f-b}\right)+O\left((\log r)^{\rho_{\log }(f)-1+\varepsilon}\right)
$$

for any $\varepsilon>0$.

If $f$ is a transcendental entire function of finite logarithmic order, then Lemma 3.1 shows that $\lambda_{\log }(f, a)+1 \leq \rho_{\log }(f)$, and Lemma 3.2 shows that $\lambda_{\log }(f, a)+1 \geq \rho_{\log }(f)$ for any finite complex constant. Thus, $\lambda_{\log }(f, a)+1=$ $\rho_{\log }(f)$ for any transcendental entire function $f$ of finite logarithmic order. Denote

$$
P(z)=z^{m} \prod_{n=0}^{\infty}\left(1-\frac{z}{z_{n}}\right)
$$

where $\left\{z_{n}\right\}$ is the zero set of $f$ and $z_{n} \neq 0$ and $m \in \mathbb{N}^{0}$. We have $\lambda_{\log }(P)+1=$ $\rho_{\log }(P)$. If $f$ is a transcendental meromorphic function of finite logarithmic order, then $f$ can be expressed in the form

$$
f=\text { const. } z^{t} \frac{P(z)}{Q(z)}=\text { const. } z^{t} \frac{\prod_{n=0}^{\infty}\left(1-\frac{z}{a_{n}}\right)}{\prod_{m=0}^{\infty}\left(1-\frac{z}{b_{m}}\right)},
$$

where $\left\{a_{n} \neq 0\right\}$ and $\left\{b_{n} \neq 0\right\}$ are the sets of zeros and poles of $f$, respectively, and $t \in \mathbb{Z}$. It is clear that $\lambda_{\log }(f, 0)=\lambda_{\log }(P)=\rho_{\log }(P)-1$ and $\lambda_{\log }(f, \infty)=$ $\lambda_{\log }(Q)=\rho_{\log }(Q)-1$.

Lemma 3.3. Let $P(z)$ be canonical product formed with the set $\left\{z_{n}\right\}, \lambda$ be the logarithmic exponent of convergence of $\left\{z_{n}\right\}$ and $C_{n}:\left|z-z_{n}\right|=\frac{1}{r_{n}^{\lambda+\varepsilon}}$ be a circle about $z_{n}$, where $r_{n}=\left|z_{n}\right|$. If $z$ lies outside of $\left\{C_{n}\right\}$ and $|z|=r>1$, then we have

$$
\log \frac{1}{|P(z)|}=O\left((\log r)^{\lambda+1+\varepsilon}\right)
$$

Proof. We write

$$
\begin{aligned}
\log \frac{1}{|P(z)|} & =\sum_{n=0}^{\infty} \log \frac{1}{\left|1-\frac{z}{z_{n}}\right|}=\sum_{2 r \leq r_{n}} \log \frac{1}{\left|1-\frac{z}{z_{n}}\right|}+\sum_{2 r \geq r_{n}} \log \frac{1}{\left|1-\frac{z}{z_{n}}\right|} \\
& =\sum_{1}+\sum_{2} .
\end{aligned}
$$


In $\sum_{2}$, if $z$ lies outside of $\left\{C_{n}\right\}$, then

$$
\log \left|\frac{z_{n}}{z-z_{n}}\right| \leq \log r_{n}^{\lambda+1+\varepsilon} \leq(\lambda+1+\varepsilon) \frac{(\log 2 r)^{\lambda+1+\varepsilon}}{\left(\log r_{n}\right)^{\lambda+\varepsilon}}
$$

Therefore,

$$
\sum_{2} \log \left|\frac{z_{n}}{z-z_{n}}\right| \leq(\lambda+1+\varepsilon)(\log 2 r)^{\lambda+1+\varepsilon} \sum_{n=0}^{\infty} \frac{1}{\left(\log r_{n}\right)^{\lambda+\varepsilon}} .
$$

In $\sum_{1}$, by using Lemma 3.1, Riemann-Stieltjes integration and L'Hospital's rule yields

$$
\begin{aligned}
\sum_{1} \log \frac{1}{\left|1-\frac{z}{z_{n}}\right|} & \leq \sum_{1} \log \frac{1}{1-\frac{r}{r_{n}}}=\int_{2 r}^{\infty} \log \frac{1}{1-r / t} d n(t) \\
& \leq \int_{2 r}^{\infty} \frac{n(t)}{t} \frac{r}{t-r} d t=O\left(\int_{2 r}^{\infty} \frac{(\log t)^{\lambda+\varepsilon}}{t^{2}} \frac{r}{1-r / t} d t\right) \\
& =O\left(r \int_{2 r}^{\infty} \frac{(\log t)^{\lambda+\varepsilon}}{t^{2}} d t\right)=O\left((\log r)^{\lambda+\varepsilon}\right) .
\end{aligned}
$$

From (7) and (8), we have the assertion by

$$
\sum_{n=0}^{\infty}\left(1 / \log r_{n}\right)^{\lambda+\varepsilon}<\infty
$$

The circles $\left\{C_{n}\right\}$ in Lemma 3.3 form an $\varepsilon$-set (see [18]). For almost all fixed $\theta$ and $r>r_{0}(\theta), z=r e^{i \theta}$ lies outside of the $\varepsilon$-set, and the set $E$ of $r$ for which the circle $|z|=r$ meets the circle of an $\varepsilon$-set has finite logarithmic measure and zero density.

Lemma 3.4 ([11], Lemma 4.1). Let $f$ be meromorphic in $\mathbb{D}$, and let $\left\{z_{n}(a)\right\}$ be the sequence of a-points of $f$, where $a \in \widehat{\mathbb{C}}$. If $\mu>0$, then

$$
\sum_{n} 1 /\left.|\log | z_{n}(a)\right|^{\mu} \quad \text { and } \int^{\infty} \frac{n\left(t, \frac{1}{f-a}\right)}{t(\log t)^{\mu+1}} d t
$$

are either simultaneously convergent or simultaneously divergent.

The following lemma is a simple analogue of Lemma 1.2.6 in [20].

Lemma 3.5. Let $f_{1}, f_{2}$ be two entire functions with no common zeros. Then for $E=f_{1} f_{2}$ we get

$$
\lambda_{\log }(E)=\max \left\{\lambda_{\log }\left(f_{1}\right), \lambda_{\log }\left(f_{2}\right)\right\} .
$$

Proof. The inequality $\max \left\{\lambda_{\log }\left(f_{1}\right), \lambda_{\log }\left(f_{2}\right)\right\} \leq \lambda_{\log }(E)$ is trivial. To prove the converse inequality, we may assume that $\lambda_{\log }(E)=\lambda \in(0, \infty)$, for otherwise there is nothing to prove. Then, for any $\varepsilon>0$, the integral

$$
\int^{\infty} \frac{n\left(t, \frac{1}{E}\right)}{t(\log t)^{\lambda+1-\varepsilon}} d t
$$


diverges by the definition of the logarithmic exponent of convergence and Lemma 3.4. Since $f_{1}$ and $f_{2}$ have no common zeros, it follows that

$$
n\left(r, \frac{1}{E}\right)=n\left(r, \frac{1}{f_{1}}\right)+n\left(r, \frac{1}{f_{2}}\right) .
$$

Therefore, at least one of the integrals

$$
\int^{\infty} \frac{n\left(t, \frac{1}{f_{1}}\right)}{t(\log t)^{\lambda+1-\varepsilon}} d t, \quad \int^{\infty} \frac{n\left(t, \frac{1}{f_{2}}\right)}{t(\log t)^{\lambda+1-\varepsilon}} d t
$$

diverges, which means that

$$
\max \left\{\lambda_{\log }\left(f_{1}\right), \lambda_{\log }\left(f_{2}\right)\right\} \geq \lambda-\varepsilon .
$$

Since $\varepsilon>0$ is arbitrary, we have the assertion.

Combining Lemma 3.5 and Lemma 3.2 together, we have the following consequence.

Corollary 3.6. Let $f_{1}, f_{2}$ be two entire functions of finite logarithmic order with no common zeros. Then for $E=f_{1} f_{2}$ we get

$$
\rho_{\log }(E)=\max \left\{\rho_{\log }\left(f_{1}\right), \rho_{\log }\left(f_{2}\right)\right\} .
$$

\section{4. $q$-Casorati determinants}

Let $f_{1}, \ldots, f_{n}$ be linearly independent entire solutions of a differential equation

$$
f^{(n)}+a_{n-1}(z) f^{(n-1)}+\cdots+a_{1}(z) f^{\prime}+a_{0}(z) f=0
$$

with entire coefficients $a_{0}(z), \ldots, a_{n-1}(z)$. Then it is well-known that the Wronskian determinant $W=W\left(f_{1}, \ldots, f_{n}\right)$ satisfies the differential equation $W^{\prime}+a_{n-1}(z) W=0$ (see [20, Proposition 1.4.8]). An analogous result is true for difference equations and a Casorati determinant (see [21, p. 86, Satz 16]). We proceed to consider the situation for $q$-difference equations recalling first the definition of a $q$-Casorati determinant (see [16]).

If $f_{1}, \ldots, f_{n}$ are meromorphic functions, then

$$
D_{q}(z)=\left|\begin{array}{cccc}
f_{1}(z) & f_{2}(z) & \cdots & f_{n}(z) \\
f_{1}(q z) & f_{2}(q z) & \cdots & f_{n}(q z) \\
\vdots & \vdots & & \vdots \\
f_{1}\left(q^{n-1} z\right) & f_{2}\left(q^{n-1} z\right) & \cdots & f_{n}\left(q^{n-1} z\right)
\end{array}\right|
$$

is called a $q$-Casorati determinant. Alternatively, we write

$$
D_{q}^{\Delta}(z)=\left|\begin{array}{cccc}
f_{1}(z) & f_{2}(z) & \cdots & f_{n}(z) \\
\Delta_{q} f_{1}(z) & \Delta_{q} f_{2}(z) & \cdots & \Delta_{q} f_{n}(z) \\
\vdots & \vdots & & \vdots \\
\Delta_{q}^{n-1} f_{1}(z) & \Delta_{q}^{n-1} f_{2}(z) & \cdots & \Delta_{q}^{n-1} f_{n}(z)
\end{array}\right|,
$$


where $\Delta_{q} f=f(q z)-f(z)$ and $\Delta_{q}^{n+1} f=\Delta_{q}^{n} f(q z)-\Delta_{q}^{n} f(z)$. By basic properties of determinants, we have $D_{q}^{\Delta}(z)=D_{q}(z)$.

If $q \in \mathbb{C} \backslash\{0.1\}$, then the $q$-Casorati determinant vanishes identically on $\mathbb{C}$ if and only if the functions $f_{1}, \ldots, f_{n}$ are linearly independent over the field of $K_{q}:=\{\gamma(z) \mid \gamma(z)=\gamma(q z), \gamma$ is meromorphic $\}$. Moreover, if $|q| \neq 1$, then this field consists of only constant functions. That is because $\gamma(z)$ is of logarithmic order one by Theorem 1.1, and $\gamma(z) \in K_{q}$ has no zeros and no poles from the equality $\gamma(z)=\gamma(q z)$, which is impossible. Hence, for $|q| \neq 1$, the linear independence of functions over $K_{q}$ is in the classical sense.

In the real line, a linear $q$-difference equation of order $n$ has a fundamental set of $n$ linearly independent solutions (see [1]). However, in the complex plane it is still an open problem to find conditions for the meromorphic coefficients under which (1) has $n$ linearly independent meromorphic solutions over $K_{q}$. Examples 3 and 4 in [5] illustrate that a linear $q$-difference equation of order two can have at least two linearly independent solutions.

Theorem 4.1. Suppose that $f_{1}, \ldots, f_{n}$ are meromorphic solutions of equation (4) and linearly independent over the field $K_{q}$. Then $D_{q}(z)$ satisfies the $q$ difference equation

$$
a_{n}(z) D_{q}(q z)+(-1)^{n+1} a_{0}(z) D_{q}(z)=0 .
$$

Proof. Since

$$
f\left(q^{n} z\right)=-\sum_{i=0}^{n-1} \frac{a_{0}(z)}{a_{n}(z)} f\left(q^{i} z\right),
$$

it follows that

$$
\begin{aligned}
D_{q}(q z) & =\left|\begin{array}{cccc}
f_{1}(q z) & f_{2}(q z) & \cdots & f_{n}(q z) \\
f_{1}\left(q^{2} z\right) & f_{2}\left(q^{2} z\right) & \cdots & f_{n}\left(q^{2} z\right) \\
\vdots & \vdots & & \vdots \\
f_{1}\left(q^{n} z\right) & f_{2}\left(q^{n} z\right) & \cdots & f_{n}\left(q^{n} z\right)
\end{array}\right| \\
& =-\left|\begin{array}{cccc}
f_{1}(q z) & \cdots & f_{n}(q z) \\
f_{1}\left(q^{2} z\right) & \cdots & f_{n}\left(q^{2} z\right) \\
\vdots & & \vdots \\
\sum_{i=0}^{n-1} \frac{a_{0}(z)}{a_{n}(z)} f_{1}\left(q^{i} z\right) & \cdots & \sum_{i=0}^{n-1} \frac{a_{0}(z)}{a_{n}(z)} f_{n}\left(q^{i} z\right)
\end{array}\right| \\
& =-\left|\begin{array}{cccc}
f_{1}(q z) & f_{2}(q z) & \cdots & f_{n}(q z) \\
f_{1}\left(q^{2} z\right) & f_{2}\left(q^{2} z\right) & \cdots & f_{n}\left(q^{2} z\right) \\
\vdots & \vdots & & \vdots \\
\frac{a_{0}(z)}{a_{n}(z)} f_{1}(z) & \frac{a_{0}(z)}{a_{n}(z)} f_{2}(z) & \cdots & \frac{a_{0}(z)}{a_{n}(z)} f_{n}(z)
\end{array}\right| \\
& =(-1)^{n} \frac{a_{0}(z)}{a_{n}(z)} D_{q}(z) .
\end{aligned}
$$

This proves the assertion. 
Next we consider the case $a_{n}(z) \equiv 1$ in (4), that is,

$$
f\left(q^{n} z\right)+a_{n-1}(z) f\left(q^{n-1} z\right)+\cdots+a_{0}(z) f(z)=0 .
$$

Theorem 4.1 and Theorem 1.1 yield the following consequence.

Corollary 4.2. Suppose that $f_{1}, \ldots, f_{n}$ are entire solutions of equation (9) and linearly independent over the field $K_{q}$. If $a_{0}(z), \ldots, a_{n}(z)$ are entire functions of finite logarithmic order, then

$$
\rho_{\log }\left(D_{q}\right)=\rho_{\log }\left(a_{0}\right)+1 .
$$

Theorem 4.3. Suppose that $f_{1}, \ldots, f_{n}$ are entire solutions of equation (9) and linearly independent over the field $K_{q}$. If $a_{0}(z), \ldots, a_{n}(z)$ are entire functions of finite logarithmic order and

$$
\rho_{\log }\left(a_{0}\right)>\max _{0<j<n}\left\{\rho_{\log }\left(a_{j}\right)\right\},
$$

then the product $E:=f_{1} \cdots f_{n}$ is of the logarithmic order of

$$
\rho_{\log }(E)=\rho_{\log }\left(a_{0}\right)+1 \text {. }
$$

Proof. Theorem 1.1 and (10) imply that every solution $f$ of the equation (9) satisfies

$$
\rho_{\log }(f)=\rho_{\log }\left(a_{0}\right)+1
$$

so that

$$
\rho_{\log }(E) \leq \rho_{\log }\left(a_{0}\right)+1 .
$$

Now dividing $D_{q}(z)$ by $E=f_{1} \cdots f_{n}$, we obtain

$$
\frac{D_{q}}{E}=\left|\begin{array}{cccc}
1 & 1 & \ldots & 1 \\
\frac{f_{1}(q z)}{f(z)} & \frac{f_{2}(q z)}{f(z)} & \ldots & \frac{f_{n}(q z)}{f(z)} \\
\vdots & \vdots & & \vdots \\
\frac{f_{1}\left(q^{n-1} z\right)}{f(z)} & \frac{f_{2}\left(q^{n-1} z\right)}{f(z)} & \ldots & \frac{f_{n}\left(q^{n-1} z\right)}{f(z)}
\end{array}\right| .
$$

By using Theorem 2.2 and (11), we have, for every $\varepsilon>0$, that

$$
m\left(r, \frac{D_{q}}{E}\right)=O\left((\log r)^{\rho_{\log }\left(a_{0}\right)+\varepsilon}\right) .
$$

By writing $D_{q}=\frac{D_{q}}{E} E$, we see that

$$
\begin{aligned}
T\left(r, D_{q}\right) & \leq m\left(r, \frac{D_{q}}{E}\right)+N\left(r, \frac{D_{q}}{E}\right)+T(r, E) \\
& \leq m\left(r, \frac{D_{q}}{E}\right)+2 T(r, E),
\end{aligned}
$$

and hence

$$
\rho_{\log }\left(D_{q}\right) \leq \max \left\{\rho_{\log }\left(a_{0}\right)+\varepsilon, \rho_{\log }(E)\right\} .
$$

According to Corollary 4.2 , we have $\rho_{\log }(E) \geq \rho_{\log }\left(a_{0}\right)+1$. This together with (12) yields the assertion. 


\section{Proof of Theorem 1.1}

First we consider homogeneous $q$-difference equations of the form (4).

Theorem 5.1. Let $a_{0}(z), \ldots, a_{n}(z)$ be meromorphic functions of finite logarithmic order such that

$$
\rho_{\log }\left(a_{i}\right)>\max _{j \neq i}\left\{\rho_{\log }\left(a_{j}\right)\right\}
$$

for some $i \in\{0, \ldots, n\}$, and let $q \in \mathbb{C} \backslash\{0\}$. If $f$ is a meromorphic solution of $(4)$, then $\rho_{\log }(f) \geq \rho_{\log }\left(a_{i}\right)$. Moreover, if $a_{0}(z), \ldots, a_{n}(z)$ are entire functions of finite logarithmic order, then $\rho_{\log }(f) \geq \rho_{\log }\left(a_{i}\right)+1$.

Proof. Let us suppose $a_{0}(z), \ldots, a_{n}(z)$ are entire functions first. We divide equation (4) by $f\left(q^{i} z\right)$ to obtain

$$
a_{i}(z)=-\sum_{\substack{j=0 \\ j \neq i}}^{n} a_{j}(z) \frac{f\left(q^{j} z\right)}{f\left(q^{i} z\right)} .
$$

Using Corollary 2.3 and (13), we have

$$
\begin{aligned}
m\left(r, a_{i}\right) & \leq \sum_{\substack{j=0 \\
j \neq i}}^{n} m\left(r, a_{j}\right)+O\left((\log r)^{\rho_{\log }(f)-1+\varepsilon}\right) \\
& \leq O\left((\log r)^{\rho_{\log }\left(a_{i}\right)-\varepsilon}\right)+O\left((\log r)^{\rho_{\log }(f)-1+\varepsilon}\right)
\end{aligned}
$$

for each $\varepsilon>0$ small enough. There is a sequence $\left(r_{n}\right)$ of points tending to infinity such that $m\left(r_{n}, a_{i}\right) \geq\left(\log r_{n}\right)^{\rho_{\log }\left(a_{i}\right)-\varepsilon / 2}$. This gives us

$$
\rho_{\log }\left(a_{i}\right)-\varepsilon / 2 \leq \rho_{\log }(f)-1+\varepsilon,
$$

where we may let $\varepsilon \rightarrow 0^{+}$.

If $a_{0}(z), \ldots, a_{n}(z)$ are meromorphic functions, then (14) implies that

$$
N\left(r, a_{i}\right) \leq \sum_{\substack{j=0 \\ j \neq i}}^{n} N\left(r, a_{j}\right)+O\left((\log r)^{\rho_{\log }(f)+\varepsilon}\right) .
$$

Together with (15), we have

$$
\rho_{\log }\left(a_{i}\right)-\varepsilon / 2 \leq \rho_{\log }(f)+\varepsilon,
$$

where we may let $\varepsilon \rightarrow 0^{+}$.

Next we consider non-homogeneous $q$-difference equations of the form

$$
\sum_{j=0}^{n} a_{j}(z) f\left(q^{j} z\right)=a_{n+1}(z) .
$$


Lemma 5.2 ([19], Lemma 3.3). Let the coefficients $a_{0}(z), \ldots, a_{n+1}(z)$ of $(16)$ be meromorphic. If $f$ is a meromorphic solution of (16), then there exists a finite constant $C>0$ such that

$$
n(r, f) \leq C\left(\sum_{j=0}^{n+1} n\left(r, a_{j}\right)+n\left(r, \frac{1}{a_{0}}\right)\right) \log r
$$

for sufficiently large values of $r$.

The following result originates from [19, Theorem 3.5], which deals with the case of the usual order of growth.

Theorem 5.3. Let $a_{0}(z), \ldots, a_{n}(z), a_{n+1}(z)$ be meromorphic functions of finite logarithmic order. Denote

$$
\rho=\max _{0 \leq j \leq n+1}\left\{\rho_{\log }\left(a_{j}\right)\right\}
$$

and let $q \in \mathbb{C} \backslash\{0\}$ be such that $|q| \neq 1$. If $f$ is a meromorphic solution of (16), then $\rho_{\log }(f) \leq \rho+1$.

Proof. According to Lemma 3.1, we deduce

$$
n\left(r, a_{j}\right)+n\left(r, 1 / a_{0}\right)=O\left((\log r)^{\rho-1+\varepsilon}\right),
$$

where $j=0, \ldots, n+1$. By using Lemma 5.2 , we have

$$
n(r, f)=O\left((\log r)^{\rho+\varepsilon}\right),
$$

which shows that

$$
N(r, f)=O\left((\log r)^{\rho+1+\varepsilon}\right) .
$$

To estimate $m(r, f)$, let us assume that the coefficients $a_{0}, \ldots, a_{n}, a_{n+1}$ are entire and $|q|>1$ at first. Following the proof of Theorem 3.5 in [19], we apply Lemma 3.3 to fix certain discs of radius $\left|z_{j}\right|^{-(\rho+\varepsilon)}$ around the zeros $z_{j}$ of $a_{n}$ such that outside of these discs, $\left|a_{n}(z)\right|>\exp \left(-(\log r)^{\rho_{\log }\left(a_{n}\right)+\varepsilon}\right)$ for sufficiently large values of $r$. Let us fix $T$ such that $f$ has no poles with modulus $|q|^{j} T$ for any $j \in \mathbb{N}$ and these circles are outside of the discs of radius $\left|z_{j}\right|^{-(\rho+\varepsilon)}$. Then

$$
\begin{aligned}
M\left(|q|^{k+n} T, f\right) & \leq \exp \left(\left(\log |q|^{k} T\right)^{\rho+\varepsilon}\right)\left(\sum_{j=k}^{k+n-1} M\left(|s|^{j} T, f\right)+1\right) \\
& \leq \exp \left(\left(\log |q|^{k} T\right)^{\rho+\varepsilon}\right)\left(\sum_{j=k}^{n+k-1} M_{j}+1\right) \\
& \leq \exp \left(\left(\log |q|^{k} T\right)^{\rho+\varepsilon}\right)(n+1) M_{k+n-1},
\end{aligned}
$$

where $M_{k}=\max _{j=0,1, \ldots, k} M\left(|q|^{j} T, f\right)+1$. Thus, for

$$
k \geq \frac{(\log (n+1))^{\frac{1}{\rho+\varepsilon}}-\log T}{\log |q|},
$$


we have

$$
M_{k+n} \leq \exp \left(A\left(\log |q|^{k} T\right)^{\rho+\varepsilon}\right) M_{k+n-1}
$$

for some $A$ and all $k \in \mathbb{N}$. Therefore,

$$
\log M_{k+n} \leq A\left(\log |q|^{k} T\right)^{\rho+\varepsilon}+\log M_{n+k-1}
$$

for large $k$. It follows that

$$
\begin{aligned}
m\left(|q|^{k+n} T, f\right) & \leq \log M\left(|q|^{k+n} T, f\right) \leq A k\left(\log |q|^{k} T\right)^{\rho+\varepsilon} \\
& =A\left(\log |q|^{k} T\right)^{\rho+\varepsilon}\left(\frac{\log \left(|q|^{k} T\right)-\log T}{\log |q|}\right) .
\end{aligned}
$$

Combining (17) and (18), we obtain

$$
T(r, f)=O\left((\log r)^{\rho+1+\varepsilon}\right)
$$

for $r=|q|^{k} T, k \in \mathbb{N}, k \rightarrow \infty$. Since $T(r, f)$ is increasing, it is now not difficult to see that the last equation also holds if $r \rightarrow \infty$ through any sequence of $r$ values. If $|q|<1$, we denote $s=1 / q$ and make a change of a variable in (16) by replacing $z$ with $s^{n} z$. This leads to a linear $s$-difference equation, corresponding to (16). This change of a variables does not interfere with the growth of the coefficients or solutions by p. 36 in [15]. The proof given above now applies to this new equation.

Finally, if the coefficients are meromorphic, we multiply away denominators, and then proceed similarly as above.

From Theorems 5.1 and 5.3, we have Theorem 1.1.

\section{First and second order equations}

We add some remarks about first and second order $q$-difference equations. We recall from [11] that if $f$ is a meromorphic function in $\mathbb{C}$ of finite logarithmic order $\rho_{\log }(f)$, then $\alpha \in \mathbb{C}$ is called a logarithmic Borel exceptional value of $f$, provided that

$$
\lambda(f, \alpha)=\limsup _{r \rightarrow \infty} \frac{n\left(r, \frac{1}{f-\alpha}\right)}{\log \log r}<\rho_{\log }(f)-1 .
$$

According to Lemma 3.2, a meromorphic function $f$ of finite logarithmic order has at most one logarithmic Borel exceptional value.

We begin with the first order $q$-difference equation

$$
f(q z)=A(z) f(z),
$$

where $A(z)$ is the finite logarithmic order function and $|q| \neq 1$. If $A(z)$ is entire and $f$ is the solution of (19), then Theorem 1.1 shows that $\rho_{\log }(f)=\rho_{\log }(A)+1$. Suppose that $A(z)$ is meromorphic, write $A(z)=\pi_{1}(z) / \pi_{2}(z)$, where $\pi_{1}(z)$ and $\pi_{2}(z)$ are canonical products formed form zeros and poles of $A(z)$, respectively. If one of $\{0, \infty\}$ is a logarithmic Borel exceptional value of $A(z)$, then according to Theorem 1.1 and Lemma 3.2, we still have $\rho_{\log }(f)=\rho_{\log }(A)+1$. Suppose 
there exists one solution $f$ satisfying $\rho_{\log }(f)<\rho_{\log }(A)+1$, then $\lambda_{\log }(A)=$ $\lambda_{\log }(A, \infty)=\rho_{\log }(A)-1$. Moreover, we have

$$
N\left(r, \frac{1}{A}\right)=T(r, A)+S(r, A) \quad \text { and } \quad N(r, A)=T(r, A)+S(r, A)
$$

by Corollary 2.3 .

We proceed with the second order $q$-difference equation

$$
f\left(q^{2} z\right)+A(z) f(q z)+B(z) f(z)=0,
$$

where $A(z), B(z)$ are entire functions of finite logarithmic order and $|q| \neq 1$. If there exist two meromorphic solutions $f_{1}, f_{2}$ of (20), linearly independent over $K_{q}$, such that the $q$-Casorati determinant of $f_{1}$ and $f_{2}$ does not vanish identically and satisfies

$$
D_{q}(q z)=B(z) D_{q}(z)
$$

By using Theorem 5.3, we have

$$
\begin{aligned}
\rho_{\log }(B)+1=\rho_{\log }\left(D_{q}\right) \leq \rho_{\log }(E) & \leq \max \left\{\rho_{\log }\left(f_{1}\right), \rho_{\log }\left(f_{2}\right)\right\} \\
& \leq \max \left\{\rho_{\log }(A), \rho_{\log }(B)\right\}+1
\end{aligned}
$$

This means that

$$
\max \left\{\rho_{\log }\left(f_{1}\right), \rho_{\log }\left(f_{2}\right)\right\}=\max \left\{\rho_{\log }(A), \rho_{\log }(B)\right\}+1 .
$$

Suppose that $\rho_{\log }(B)<\rho_{\log }(A)$ and $f_{i}(i=1,2)$ are entire functions. According to Lemma 3.2 , it follows that

$$
\begin{aligned}
\rho_{\log }(A)+1 & =\rho_{\log }\left(f_{1}\right)=\lambda_{\log }\left(f_{1}\right)+1 \leq \lambda_{\log }(E)+1 \\
& =\rho_{\log }(E)=\rho_{\log }(B)+1,
\end{aligned}
$$

which is impossible. Now we apply the reasoning of above, and obtain the following theorem.

Theorem 6.1. If $f_{1}$ and $f_{2}$ are solutions of (20), linearly independent over $K_{q}$, then at least one of $f_{1}$ and $f_{2}$ satisfies

$$
\rho_{\log }\left(f_{i}\right)=\max \left\{\rho_{\log }(A), \rho_{\log }(B)\right\}+1,
$$

where $i=1$ or $i=2$. Moreover, if there exists two entire linearly independent solutions over $K_{q}$ satisfying $(20)$, then $\rho_{\log }(B) \geq \rho_{\log }(A)$.

\section{Examples}

Suppose that $A(z)$ is an entire function of finite logarithmic order $\rho_{\log }(A)$, and let $q \in \mathbb{C} \backslash\{0\}$. If $|q| \neq 1$, then every meromorphic solution $f$ of (19) satisfies $\rho_{\log }(f)=\rho_{\log }(A)+1$ by Theorem 1.1. This result is useful in the following examples. 
Example 1. Let $q \in \mathbb{C}$ be such that $0<|q|<1$. Then the $q$-gamma function is defined by

$$
\Gamma_{q}(x):=\frac{(q ; q)_{\infty}}{\left(q^{x} ; q\right)_{\infty}}(1-q)^{1-x}
$$

where $(a ; q)_{\infty}=\Pi_{k=0}^{\infty}\left(1-a q^{k}\right)$ (see [2, p. 493]). Here we take the principal values of $q^{x}$ and $(1-q)^{x}$. Thus, $\Gamma_{q}(x)$ is a meromorphic function with poles at $x=-n \pm 2 \pi i k / \log q$, where $k$ and $n$ are non-negative integers. Define

$$
\gamma_{q}(z):=(1-q)^{x-1} \Gamma_{q}(x), \quad z=q^{x},
$$

and $\gamma_{q}(0):=(q ; q)_{\infty}$. Then $\gamma_{q}(z)$ is a meromorphic function of order zero with no zeros, having its poles at $\left\{q^{-k}\right\}_{k=0}^{\infty}$, see [3]. Moreover, $\gamma_{q}(z)$ satisfies the $q$-difference equation (19) with $A(z)=1-z$. Hence, in fact, $\gamma_{q}(z)$ has logarithmic order 2 .

Example 2. Given any positive integer $m$ it is easy to construct an entire function of logarithmic order $m$. Denote $W_{1}(z)=1-z$ and $W_{2}(z)=$ $\prod_{n=1}^{\infty} W_{1}\left(z / q^{n}\right)$, where $|q|>1$. In fact, $W_{2}(z)=\prod_{n=1}^{\infty}\left(1-\frac{z}{q^{n}}\right)$, which clearly converges in compact subsets of $\mathbb{C}$, and hence is entire. It is clear that $W_{2}(z)$ satisfies

$$
W_{2}(q z)=(1-z) W_{2}(z)=W_{1}(z) W_{2}(z) .
$$

Therefore, $W_{2}(z)$ has logarithmic order 2. Further, the entire function $W_{3}(z)=$ $\prod_{n=1}^{\infty} W_{2}\left(z / q^{n}\right)$ satisfies

$$
W_{3}(q z)=W_{2}(z) W_{3}(z)
$$

and hence it has logarithmic order 3. Proceeding inductively, for any positive integer $m$, the entire function

$$
W_{m}(z)=\prod_{n=1}^{\infty} W_{m-1}\left(z / q^{n}\right)
$$

satisfies the $q$-difference equation

$$
W_{m}(q z)=W_{m-1}(z) W_{m}(z)
$$

and has logarithmic order $\rho_{\log }\left(W_{m}\right)=m$.

Example 3. $q$-exponential functions are $q$-analogues of the exponential function $e^{z}$, and are denoted by

$$
E_{q}(z)=(-z, q)_{\infty} \text { and } \quad e_{q}(z)=1 /(z, q)_{\infty} .
$$

We have $e_{q}(z) E_{q}(-z)=1$ (see [14, p. 11]). Both of $E_{q}(z)$ and $e_{q}(z)$ satisfy (19) with $A=1+z$ and $A(z)=1 /(1-z)$, respectively. Hence $E_{q}(z)$ and $e_{q}(z)$ have logarithmic order 2. Moreover, it is clear that

$$
E_{q}\left(z^{2}\right)=E_{\sqrt{q}}(z) E_{-\sqrt{q}}(z) .
$$

Since $E_{\sqrt{q}}(z)$ and $E_{-\sqrt{q}}(z)$ have no common zeros, Corollary 3.6 implies that $E_{q}\left(z^{2}\right)$ is of logarithmic order two, the same order as for $E_{q}(z)$. Proceeding 
inductively, $E_{q}\left(z^{n}\right)$ has logarithmic order 2, where $n$ is any integer. In contrast, the functions $e^{z^{n}}$ and $e^{z}$ have different orders, if $n \neq 1$.

Acknowledgements. The author would like to thank his supervisor Janne Heittokangas for making suggestions to improve the paper.

\section{References}

[1] M. H. Abu Risha, M. H. Annaby, M. E. H. Ismail, and Z. S. Mansour, Linear q-difference equations, Z. Anal. Anwend. 26 (2007), no. 4, 481-494.

[2] G. E. Andrews, R. Asker, and R. Roy, Special Functions, Cambridge University press, 1999.

[3] D. C. Barnett, R. G. Halburd, W. Morgan, and R. J. Korhonen, Nevanlinna theory for the q-difference operator and meromorphic solutions of q-difference equations, Proc. Roy. Soc. Edinburgh Sect. A 137 (2007), no. 3., 457-474.

[4] C. Berg and H. L. Pedersen, Logarithmic order and type of indeterminate moment problems, With an appendix by Walter Hayman, Difference equations, special functions and orthogonal polynomials, 51-79, World Sci. Publ., Hackensack, NJ, 2007.

[5] W. Bergweiler and W. K. Hayman, Zeros of solutions of a functional equation, Comput. Methods Funct. Theory 3 (2003), no. 1, 55-78.

[6] W. Bergweiler, K. Ishizaki, and N. Yanagihara, Meromorphic solutions of some functional equations, Methods Appl. Anal. 5 (1998), no. 3, 248-258.

[7] - Growth of meromorphic solutions of some functional equations, I. Aequationes Math. 63 (2002), no. 1-2, 140-151.

[8] B.-Q. Chen and Z.-X. Chen, Meromorphic solutions of some q-difference equations, Bull. Korean Math. Soc. 48 (2011), no. 6, 1303-1314.

[9] B.-Q. Chen, Z.-X. Chen, and S. Li, Properties on solutions of some q-difference equations, Acta Math. Sin. (Engl. Ser.) 26 (2010), no. 10, 1877-1886.

[10] Y.-M. Chiang and S. J. Feng, On the Nevanlinna characteristic of $f(z+\eta)$ and difference equations in the complex plane, Ramanujan J. 16 (2008), no. 1, 105-129.

[11] Peter T.-Y. Chern, On meromorphic functions with finite logarithmic order, Trans. Amer. Math. Soc. 358 (2006), no. 2, 473-489.

[12] J. Clunie, On integral functions having prescribed asymptotic growth, Canad. J. Math. 17 (1965), 396-404.

[13] J. Clunie and T. Kövari, On integral functions having prescribed asymptotic growth. II, Canad. J. Math. 20 (1968), 7-20.

[14] G. Gasper and M. Rahman, Basic Hypergeometric Series, Second Edition, Cambridge, Cambridge Univ. Press 2004.

[15] A. A. Goldberg and I. V. Ostrovskii, Value distribution of meromorphic functions, Translated from the 1970 Russian original by Mikhail Ostrovskii. With an appendix by Alexandre Eremenko and James K. Langley.Translations of Mathematical Monographs, 236. American Mathematical Society, Providence, RI, 2008.

[16] R. Halburd, R. Korhonen, and K. Tohge, Holomorphic curves with shift-invariant hyperplane preimages, arXiv:0903.3236v1.

[17] W. K. Hayman, Meromorphic Functions, Clarendon Press, Oxford, 1964.

[18] Slowly growing integral and subharmonic functions, Comment. Math. Helv. 34 (1960), 75-84.

[19] J. Heittokangas, I. Laine, J. Rieppo, and D.-G. Yang, Meromorphic solutions of some linear functional equations, Aequations Math. 60 (2000), no. 1-2, 148-166.

[20] I. Laine, Nevanlinna Theory and Complex Differential Equations, Walter de Gruyter, Berlin-New York, 1993. 
[21] H. Meschkowski, Differenzengleichungen, Studia Mathematica, Bd. XIV Vandenhoeck and Ruprecht, Göttingen 1959.

TAiyuan University of Technology

Department of Mathematics

030024, Taiyuan, Shanxi, P. R. China

AND

University of EAstern Finland

Department of Physics and Mathematics

P. O. Box 111, 80101 Joensuu, Finland

E-mail address: zhitaowen@gmail.com 